\title{
FRIEDRICH VON HARDENBERG (NOVALIS) ET JEAN-FRANÇOIS D'AUBUISSON DE VOISINS À LA BERGAKADEMIE DE FREIBERG
}

\author{
UN EXEMPLE DE TRANSFERT CULTUREL À LA FIN DU XVII' SIËCLE *
}

Né le 2 mai 1772 à Oberwiederstedt (Saxe), Frédéric de Hardenberg s'éteint de consomption le 25 mars 1801 à Weissenfels, âgé de vingt-neuf ans à peine. Au cours de cette existence météorique, il devait se livrer à une véritable apologie de l'activité (Tätigkeit) ${ }^{1}$. Trois lieux ont marqué ses années d'apprentissage : l'université, l'Académie des mines (Bergakademie) et l'administration des salines.

Il convient de souligner le rôle fondamental de l'université dans sa première formation (1790-1794) et, au-delà d'un cas singulier, des universités allemandes - fortement dispersées, comme l'on sait - , comme lieu de formation du savoir ${ }^{2}$. C'est, en effet, du sein de l'université que se sont affirmées trois figures majeures du savoir et du pouvoir en Allemagne : le pasteur, le professeur et le fonctionnaire.

Hardenberg fréquente successivement les universités de Iéna, Leipzig et Wittenberg, où il suit les cours de philosophie de Reinhold ${ }^{3}$, les cours d'histoire de Schiller et, vraisemblablement, les cours de mathématiques de Hindenburg ${ }^{4}$ - avant de passer et de réussir son examen d'État en droit à Wittenberg (14 juin 1794).

* M. le professeur Jean-Louis VieillaRd-Baron, Directeur du C.R.D.H.M. (C.N.R.S., Université de Poitiers) a bien voulu accueillir et soutenir cette recherche. Qu'il en soit ici bien vivement remercié.

1. Dans les pages qui suivent, nous citons les cuvres complètes de Hardenberg-Novalis dans l'édition de référence : Richard SAmUEL, Hans-Joachim MXHL, Gerhard Schulz, Hrsg., Novalis-Schriften, Stuttgart, W. Kohlhammer, 1960-1988 (5 tomes parus), cité par la suite comme HKA, suivi du tome et de la page.

Le statut de la Tatigkeit apparaît dans trois lettres qui font référence, cf. HKA, IV, p. 90, $140,287$.

2. Norbert Elias, Uber den Prozess der Zivilisation. Wandlungen des Verhaltens in den westlichen Obenschichten des Abendlandes, Francfort-sur-le-Main, 1939, 1969, trad. franç. La Civilisation des moeurs, Paris, Calmann-Lévy, 1973, p. 44.

3. Sur la présence de Platon dans l'idéalisme allemand, cf. Jean-Louis Vieillard-Baron, Platon et l'idéalisme allemand (1770-1830), Paris, Beauchesne, 1979 ; ID., Platonisme et interprétation de Platon d l'époque modeme, Paris, Vrin, 1988.

4. Cf. HKA, IV, p. 97 ; Martin Dyck, Novalis and Mathematics, Chapel Hill, The Univer-

Revue de synthèse : IV S. Nº 1-2, janv.-juin 1992. 
Hors les murs de l'université et de l'Académie ${ }^{5}$, mais dans l'intervalle ménagé entre ces deux institutions, vont se développer et s'affirmer les écoles techniques dont l'un des exemples majeurs sera la Bergakademie. Du $1^{\text {er }}$ décembre 1797 à la Pentecôte 1799, Hardenberg étudie dans ce pôle d'attraction scientifique et technique que fut pour toute l'Europe la Bergakademie de Freiberg. Elle est le lieu :

- d'une rencontre avec le Français d'Aubuisson;

- d'une pratique des arts de la mine (cf. les Salinenschriften ${ }^{6}$ );

- d'une écriture : de nombreux écrits verront le jour à Freiberg, dont le célèbre Répertoire général (Das Allgemeine Brouillon)?

Au sortir de l'université s'était posé de façon aiguë le problème de la vocation (Beruf), du métier et de l'activité (Tätigkeit). Hardenberg aurait dû être placé - des tractations ont eu lieu en ce sens - au service de l'État prussien ${ }^{8}$. Les négociations ayant traîné, il dirigea ses pas vers l'administration des salines.

C'est au début de l'année 1785 que Henri Ulrich Erasme de Hardenberg (1738-1814) ${ }^{9}$, père de Frédéric de Hardenberg, quitte son domaine d'Oberwiederstedt pour succéder à Léopold de Beust ${ }^{10}$ au poste de directeur des salines de Artern, Kösen et Dürrenberg ${ }^{10}$. L'exploitation des

sity of North Carolina Press, 1960, p. 27-31; Kleist verra en Hindenburg un « instituteur de l'humanité » (Lehrer der Menschheit), in Heinrich von KLEIST, Sämtliche Werke und Briefe II, Munich, Carl Hanser, 1977, p. 656, lettre du 3-6-1801 à Wilhelmine von Zenge.

5. Immanuel KANT, Der Streit der Fakultaten, Königsberg, 1798, trad. franç. d'Alain Renaut, in Euvres philosophiques, Paris, Gallimard, 1985, t. III, p. 813-815 et 826-827.

6. HKA, V, p. 27-124.

7. HKA, III, p. 242-478.

8. Pour ce qui est des aspects biographiques, on se reportera à August Coelestin Just, " Friedrich von Hardenberg ", 1805, in HKA, IV, p. 536-550; G. ScHULZ, « Die Berufslaufbahn Friedrich von Hardenbergs (Novalis) ", Jahrbuch der deutschen Schillergesellschaft, 7 , 1963, p. 253-312, repris in G. Schulz, Hrsg., Novalis-Beiträge zu Werk und Persönlichkeit Friedrich von Hardenbergs, Darmstadt, Wissenschaftliche Buchgesellschaft, 1970, 1986, p. 283-356; HKA, V, p. 366-404.

9. Etudiant en droit et en arts des mines à Göttingen, sa dissertation, soutenue en 1760 , est intitulée : De Libertinitate et De Judiciis Capitalibus Veterum Germanorum. Il sera tuteur de Fichte à partir de 1774.

10. Sur la problématique des salines en général, cf. Jean-Claude HocqueT, Le Sel et le pouvoir. De l'An mil à la Révolution française, Paris, A. Michel, 1984 ; ID., Le Sel de la terre, Paris, Ed. Du May, 1989, p. 74-101 ; Hans-Henning WALTER, Zur Entwicklung der Siedesalzgewinnung in Deutschland von 1500 bis 1900 unter besonderer Berücksichtigung chemischtechnologischer Probleme, Freiberg, Veröffentlichungen des wissenschaftlichen Informationszentrums der Bergakademie Freiberg, 1985.

Sur les trois salines ci-dessus citées, of. H.-H. WALTER, Historische Beiträge zur Kyffhouserlandschaft. 2000 Jahre Salzproduktion am Kyfhäuser. Geschichte der Salinen Frankenhausen, Auleben und Artern, Bad Frankenhausen, Kreisheimatmuseum Bad Frankenhausen, 1986; Johannes MAGer, Die Saline Kösen. Ein geschichtlicher Abriss, Bad Kösen, Museen der Stadt Bad Kösen, 1987; Johannes MAGER, Lutz ToEPFER, Die Saline Dürrenberg, Bad Dürrenberg, Rat der Stadt Dürrenberg, 1984. 
salines permettait alors à la Saxe de se procurer, sans importations, du sel en suffisance ${ }^{\mathrm{Il}}$.

$\mathrm{Si}$, à l'époque, Hardenberg ne montre que peu d'intérêt pour les nouvelles attributions de son père, il deviendra ensuite (après avoir été greffier à Tennstedt), aspirant auprès de la direction des salines de Weissenfels (du 5 février 1796 au $1^{\text {er }}$ décembre 1797). Le 7 décembre 1799, il occupera le poste d'assesseur des salines; enfin, le 6 décembre 1800, celui de bailli surnuméraire dans le district de Thuringe.

Cette activité pratique se double bientôt d'une activité d'écriture. Car ce n'est pas là le moindre paradoxe : les publications poétiques de Hardenberg coïncident, pour l'essentiel, avec les périodes d'activité scientifique et technique les plus intenses (1798-1800). Il convient cependant de préciser quelque peu le statut de l'écriture chez Hardenberg. S'il a beaucoup lu et écrit, il n'a que peu publié. De son vivant, seuls six ensembles de textes ont paru dans des revues:

1. Un poème de jeunesse, Plaintes d'un jeune homme (Klagen eines Jünglings), dans le Neuer Teutscher Merkur, en avril 1791;

2. En 1798, dans les Annales de la monarchie prussienne (Jahrbücher der preussischen Monarchie) : a) 8 poèmes sous le titre : Fleurs (Blumen), juin 1798; b) 43 fragments sous le titre : Foi et Amour ou le Roi et la Reine (Glauben und Liebe oder der König und die Königin);

3. De 1798 à 1800 , dans l'Athénée (Athenäum) : a) 114 fragments sous le titre : Pollens (Blütenstaub), mai 1798, sous le pseudonyme de Novalis ${ }^{12}$; b) 12 fragments anonymes insérés dans les fragments de Frédéric Schlegel, juin 1798; c) Les Hymnes à la nuit (Hymnen an die Nacht), Saint-Michel 1800.

Derrière ces quelques textes publiés, épars, affleure une masse d'ébauches, de notes, de mémoires où se déploie une écriture discontinue et feuilletée à la fois. À tel point que l'on pourrait, en empruntant à la géologie cette métaphore, parler de véritables chaos textuels.

Les éditions critiques, depuis $1802^{13}$, ont tenté de recueillir ces textes en tous genres. Or ces éditions, depuis près de deux siècles, ont été, il

11. Sur l'axiome de fermeture, cf. Johann Gottlieb Fichr, Der geschlossene Handelsstaat, Tubingen, 1800, trad. franç. de Daniel Schulthess, Lausanne, L'Äge d'homme, 1980.

12. HKA, IV, p. 251, lettre du 24-2-1798 à August Wilhelm Schlegel.

13. Dans l'ordre chronologique : celles de Friedrich Schlegel et Ludwig Tieck $(1802,1805$, 1815,1826 , 1837), Ludwig Tieck et Eduard von Bulow (1846), Emst Heilborn (1901), Jakob Minor (1907), Paul Kluckhohn (1929), Emst Kamnitzer (1929), Ewald Wasmuth (19531957), Paul Kluckhohn et Richard Samuel (1960-1988). 
faut bien en convenir, très largement tributaires de l'histoire mouvementée du Nachlass de Hardenberg ${ }^{14}$.

Pour prendre deux exemples significatifs : nous ne disposons d'une édition scientifique du Répertoire général que depuis $1963^{15}$; quant aux Écrits des salines (Salinenschriften) ${ }^{16}$, que l'on pensait à tout jamais disparus, ils ont été retrouvés en 1983 dans les fonds de la Biblioteka Jagiellonska (Cracovie) par les soins de Hans-Joachim Mähl.

C'est dire que l'édition critique et scientifique des textes de Hardenberg n'est pas une donnée, d'emblée disponible, mais qu'elle est, depuis près de deux siècles, en voie de constitution. Et si quelques textes nous font encore défaut (en particulier ses papiers scientifiques en possession de Johann Wilhelm Ritter ${ }^{17}$ ), nous sommes bien près de toucher au but.

Laissant de côté la période universitaire de Hardenberg, nous nous consacrons ici, d'une part, à sa rencontre avec d'Aubuisson, d'autre part, au transfert culturel mathématiques/géologie entre la France et l'Allemagne à l'extrême fin du xuIII' siècle $^{18}$.

Hardenberg étudie, avons-nous dit, à la Bergakademie de Freiberg de décembre 1797 à la Pentecôte 1799, afin de parfaire ses connaissances en chimie et en mathématiques ${ }^{19}$.

\section{I. - FREIBERG : LA BERGAKADEMIE}

La ville de Freiberg, située au pied des Monts Métallifères (Erzgebirge), comptait, vers la fin du xvir siècle, quelque 9400 habitants, dont plus de 5000 employés au service des mines dans le district. Vers 1800 , environ 240 mines étaient exploitées. La région présente un aspect quelque peu

14. Daniel LANCEREau, «Sur l'édition critique des cuvres de Novalis ", Etudes germaniques, Paris, juil.-sept. 1989, p. 304-308.

15. H.-J. MXHL, "Novalis und Plotin. Untersuchungen zu einer neuen Edition und Interpretation des "Allgemeinen Brouillon" ", Jahrbuch des Freien Deutschen Hochstifts, Tubingen, 1963, p. 139-250, repris in G. Schulz, Hrsg., op. cit. supra, n. 8, p. 357-423.

16. Soit 410 pages manuscrites, qui seront publiées intégralement in HKA, VI, à paraître.

17. HKA, III, p. XIV.

18. Michel ESPAGNE, Michaell WERNER, «Transferts culturels franco-allemands », numéro spécial de la Revue de synthèse, Paris, 2, avril-juin 1988.

19. La demande présentée par son père date du 31 octobre 1797, in HKA, IV, p. 611. Le $1^{\text {ex }}$ décembre 1797, Hardenberg se met en route pour rejoindre la Bergakademie. II s'arrêtera en chemin à Leipzig pour une première rencontre avec Schelling. Cf. Jean-Francois MarQUET, Liberté et existence. Etude sur la formation de la philosophie de Schelling, Paris, Gallimard, 1973 ; ID., "Schelling et la philosophie de la nature ", in Antoine FAIVRE, Rolf Christian Zimmermann, Epochen der Naturmystik, Berlin, Erich Schmidt, 1979, p. 426-444. 
triste : le pays environnant est nu et découvert; seuls les bois de sapins des environs viennent briser cette monotonie. Le climat est sec et froid; si froid, qu'en 1799 la température s'abaissa jusqu'à moins $27^{\circ}$. La neige recouvre la terre pendant deux à trois mois. Le printemps est de courte durée, ou inexistant ; les récoltes, tardives. Le soleil d'été donne des jours longs, mais peu chauds. Tout autour de la ville, mais principalement au sud, se trouvent les gîtes minéraux : antimoine, argent, arsenic, cuivre, cobalt, fer, manganèse, molybdène, plomb, zinc, etc. Le district des mines forme un cercle d'environ une lieue et demie de rayon. Jamais endroit n'a été plus propice à l'établissement d'une école des mines. Freiberg a su offrir une conjonction heureuse de circonstances favorables :

« Tout autour de la ville [...] on trouve plus de cent mines toutes exploitées dans toutes les règles de l'art [...]. Dans le court espace d'une année ces cent mines peuvent présenter un plus grand nombre de cas particuliers que dans d'autres pays on en verrait en vingt ans dans une étendue de plusieurs milliers de lieues carrées [...] des professeurs savants et expérimentés sont en ville; les exemples sont à une demi-lieue de distance $" 20$.

La Bergakademie a été fondée en 1765 (le grand-oncle de Hardenberg, Friedrich Anton von Heynitz, fut l'un de ses cofondateurs); les cours y ont débuté à la Pentecôte 1766. Cette institution devait répondre à une double préoccupation, à une double exigence :

- le recul, puis le déclin, de l'extraction minière, source de richesses pour les princes saxons. L'extraction de l'argent avait commencé au $\mathrm{XII}^{e}$ siècle et connu son apogée dans la deuxième moitié du $\mathrm{xv}^{e}$ siècle, avant de décliner;

- les difficultés économiques de la Saxe après la guerre de Sept Ans (1756-1763).

On peut considérer que, dès les années 1790 , le pari est pour ainsi dire gagné : non seulement l'extraction est en hausse, mais Freiberg, siège de la Bergakademie, devient un pôle d'attraction scientifique et technique ${ }^{\mathbf{2 1}}$.

Freiberg, devenue célèbre, renouvelle, aux dires de Cuvier, « le spectacle des premières universités du Moyen Âge ${ }^{22}$ : elle draine des élèves de tous les horizons géographiques: Portugal, Espagne, Italie, Angle-

20. Jean-François Daubuisson, Des mines de Freiberg en Saxe et de leur exploitation, Leipzig, 1802, t. II, p. 244, 246. Les renseignements qui précèdent sont tirés de cet ouvrage.

21. G. Schulz, op. cit. supra n. 8, p. 298-303; Joseph Ben-Davi, The Scientist's Role in Society. A Comparative Study, Englewood Cliffs, N.J. Prentice-Hall, 1971, p. 111 ; W.V. FaRRAR, «Science and the German University System (1790-1850) 》, in The Emergence of Science in Western Europe, éd. Maurice Crosland, Londres et Basingstoke, The Mac Millan Press Ltd, 1975, p. 179, 182-183.

22. Cité in A. Farvre, "Entre l'Aufklärung et le romantisme : A. G. Werner ou la " géognosie " ", Les érudes philosophiques, 1, 1977, p. 41-52. 
terre, Suède, Danemark et Norvège, Pologne, Russie, Allemagne, etc. Quelques noms célèbres sont passés par la Bergakademie : Baader, A. von Humboldt, Herder fils, Mohs, Steffens, Watt fils, Brochant de Villiers, d'Aubuisson, etc. ${ }^{23}$. Du temps de Hardenberg, elle comptait 50 élèves environ. Pour être admis, l'élève devait avoir entre seize et vingt-six ans; le cursus était de trois ans. Chaque année, avant Pâques, avait lieu l'examen public en présence des conseillers et officiers des mines.

Il existait deux classes d'étudiants :

- les boursiers, astreints à tenir un journal circonstancié de leurs études et activités ;

- les étudiants payants.

Un de ces étudiants payants fut précisément Hardenberg ${ }^{24}$; il est admis à la Bergakademie à l'âge de vingt-cinq ans, soit à la limite haute; de plus, son parcours est singulier, son cursus universitaire allant non des mines au droit, mais du droit aux mines.

À la Bergakademie étaient enseignées toutes les sciences qui, à un titre ou à un autre, entretenaient quelque rapport que ce soit avec l'art des mines : exploitation des mines, géologie, minéralogie, métallurgie, mécanique, chimie, physique, mathématiques, droit, etc. C'est pourquoi elle s'était assurée de la collaboration de professeurs éminents. Nous en retiendrons quatre, utiles pour notre propos :

- J.F.W. von Charpentier : il a occupé la chaire de mathématiques et physique jusqu'en 1784. Il est l'auteur d'une Mineralogische Geographie, avec carte pétrographique en huit couleurs ${ }^{25}$. Charpentier était le père de la seconde fiancée de Hardenberg : Julie von Charpentier.

- J.F. Lempe : depuis 1785 , successeur de Charpentier à la chaire de mathématiques et physique, il est l'éditeur du Magazin für Bergbaukunde $^{26}$.

- A.W. Lampadius (de même âge que Hardenberg) : à Freiberg depuis 1794, défenseur de la nouvelle chimie lavoisienne (anti-phlogistique), il découvre, en 1796, le sulfure de carbone et construit, en 1797, le premier laboratoire au monde dans une école supérieure ${ }^{27}$.

23. Abraham Gottlob Werner. Gedenkschrift aus Anlass der Wiederkehr seines Todestages nach 150 Jahren am 30. Juni 1967, hrsg. vom Rektor der Bergakademie Freiberg, Leipzig, VEB Deutscher Verlag fur Grundstoffindustrie, 1967, p. 164-166. Cf. aussi la somme de Roger Ayrault, La Genèse du romantisme allemand, Paris, Aubier, 1961-1976, 4 vol.

24. Friedrich von Hardenberg (Novalis). Studierender der Bergakademie Freiberg Dezember 1797 bis Mai 1799. Zur 200. Wiederkehr seines Geburtstages am 2. Mai 1972, Freiberg (Sachs.), Veröfientlichungen des wissenschaftichen Informationszentrums der Bergakademie Freiberg, 1972.

25. Sur Charpentier, cf. Allgemeine Deutsche Biographie, Leipzig, 1876, t. IV, p. 105-108.

26. Sur Lempe, cf. Moll's Annalen der Berg- und Hâttenkunde, Salzburg, 1802, Bd 1, p. 216-221; op. cit. supra n. 25, t. XVII, p. 240-241.

27. Sur Lampadius, cf. op. cit. supra n. 25, t. XVI, p. 578-579. 
- A.G. Werner : après avoir étudié à Freiberg en 1769, il se rend, en 1771, à Leipzig pour y étudier le droit et les sciences. En 1774, il fait paraître son livre, Des caractères extérieurs des fossiles et, l'année suivante, obtient la chaire de minéralogie et science des mines ${ }^{28}$.

Le programme des cours entre Pâques 1798 et Pâques 1799 est donné dans le tableau comparatif Freiberg/Paris ci-dessous (p. 116). L'étudiant " peut suivre les cours de divers professeurs, en payant des honoraires fixés, et qu'on ne peut outrepasser. Si un étranger désirait avoir un cours particulier à lui seul, il fallait qu'il s'arrangeât de gré à gré avec les Professeurs ${ }^{29}$. Les cours publics pouvaient donc être répétés sous forme de cours particuliers. D'Aubuisson note une certaine prolixité au niveau des cours : les professeurs s'arrêtent sur des considérations évidentes. Les manuels allemands n'ont ni la clarté ni la rapidité d'exposition des ouvrages français; sans doute d'Aubuisson songe-t-il ici aux manuels de mathématiques en particulier.

Quant à l'auditoire, il est composé « de jeunes gens destinés à occuper des emplois d'abord peu relevés dans les mines de la Saxe " et leurs connaissances sont " très bornées " ${ }^{30}$. Aux cours s'ajoutait la fréquentation de la bibliothèque; à la fréquentation de la bibliothèque, l'étude pratique : visite des mines, examen et utilisation des collections de minéraux. Enfin, il existait la possibilité de contacts personnels avec la maison Charpentier et Werner (ce que feront, tour à tour, Hardenberg et d'Aubuisson).

Hardenberg a été introduit dans la famille Charpentier par son ami Thielmann (qui avait épousé une fille Charpentier) et il suivra dès Pâques 1798 les cours de Werner (à la différence de d'Aubuisson qui n'y assiste qu'en 1800 et 1801 ).

À relire les lettres de Hardenberg à Freiberg, on décèle une différence de ton entre le début et la fin du séjour. Une inflexion, une ponctuation intervient. Dans les premiers mois, Hardenberg éprouve un sentiment de vide, d'isolement, d'éloignement, de désordre, d'étrangeté. C'est, rappelons-le, le 24 février 1798 qu'il se donne, aux fins de publication, le pseudonyme de Novalis ${ }^{31}$. Dans la deuxième moitié du séjour, les accents se déplacent; passe alors au premier plan la volonté d'apprendre et de mettre ce savoir au service des salines. Un double obstacle semble donc avoir été surmonté :

- l'incertitude extrême où l'avait laissé l'existence (après la mort de sa première fiancée, Sophie von Kühn, en mars 1797);

28. Sur Werner, cf. Abraham Gottlob Werner. Gedenkschrift..., op. cit. supra n. 23.

29. Cf. J.-F. Daubusson, op. cit. supra n. 20, t. II, p. 242-243 (souligné par nous).

30. Annales de chimie, Paris, t. 69, 1809, p. 231, 238.

31. Cf. la lettre de Hardenberg à August Wilhelm Schlegel du 24-2-1798, in HKA, IV, p. 251. 

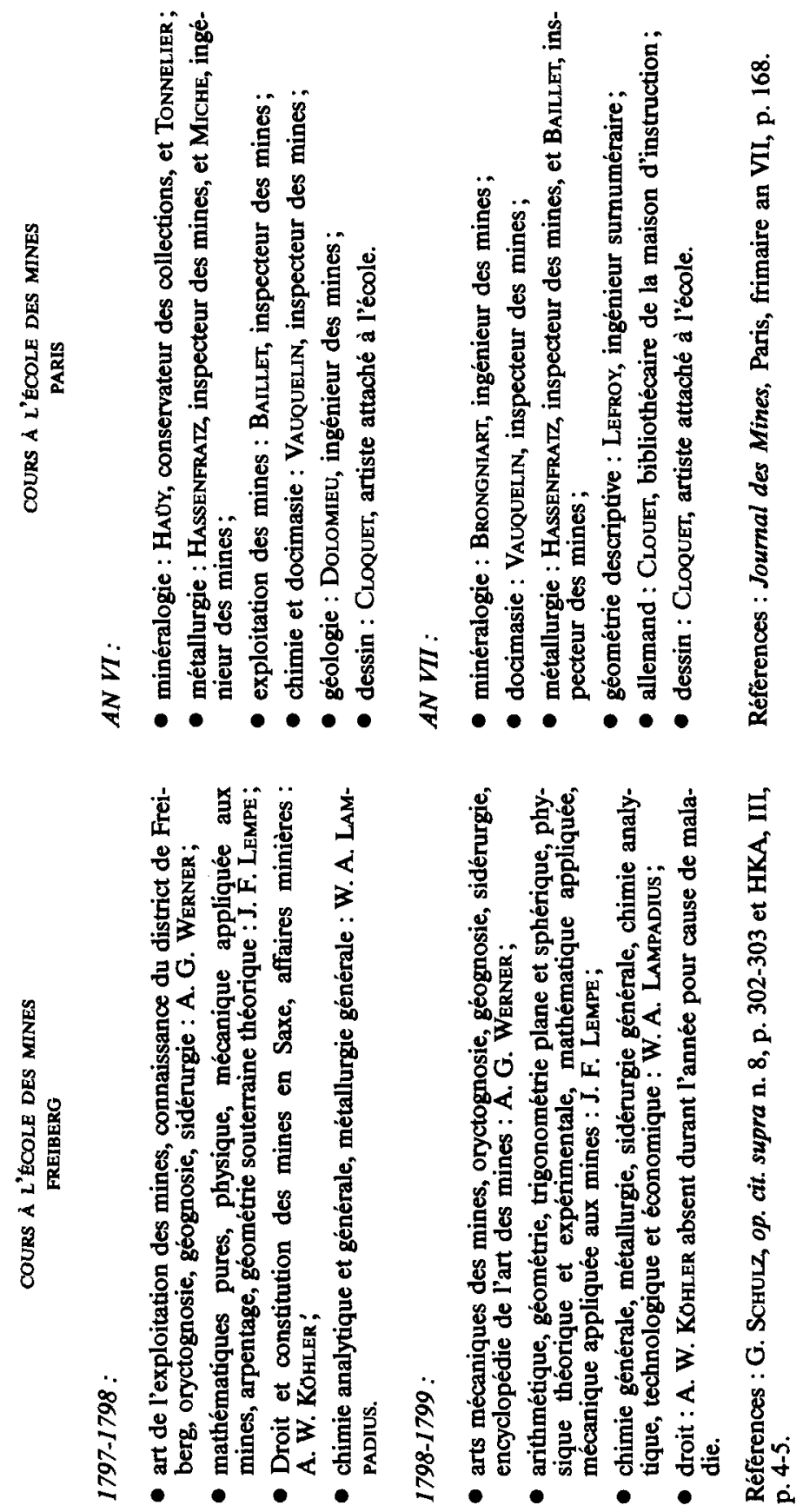
- l'empirique et l'analytique du savoir (cf. les lettres du 12 janvier 1798 et du 24 février 1798).

Ce revirement a été rendu possible, d'une part, par les contacts plus étroits avec la maison Charpentier (le poème Der Fremdling est dédié, en janvier 1798, à Mme Charpentier ; en décembre 1798 auront lieu les fiançailles avec Julie von Charpentier) et, d'autre part, par la rencontre avec son maître, le grand systématicien Werner (qui lui redonne le sens de la terre et de la Tätigkeit).

D'Aubuisson, quant à lui ${ }^{32}$, avait étudié à Sorèze ${ }^{33}$ de 1779 à 1786 et s'était particulièrement distingué dans les sciences pures et appliquées (arithmétique, géométrie, algèbre, histoire naturelle, mécanique, physique, astronomie, calcul infinitésimal) ${ }^{34}$. Après avoir étudié le droit à Toulouse, il fut aspirant d'artillerie à Metz en $1789^{35}$. Il émigra le 22 septembre 1791, passa d'abord en Espagne, avant d'être envoyé en Allemagne. Il fit la campagne de 1792 (Champagne) à l'armée des Princes, frères du roi Louis XVI, dans son corps ; puis passa à la compagnie noble d'artillerie du prince de Condé, où il participa aux campagnes de 1793 à $1797^{36}$. La paix de Campo-Formio une fois signée (18 octobre 1797), il se retira à Freiberg, vraisemblablement le 25 novembre ${ }^{37}$, soit, à quelques jours près, aux mêmes dates que Hardenberg (qui arrivera, lui, le $1^{\text {er }}$ décembre 1797).

À son arrivée, d'Aubuisson est accueilli par Charpentier et Werner. Or les relations entre les deux hommes n'étaient pas des plus sereines. Charpentier, dira d'Aubuisson, était " entièrement opposé à Werner sous tous

32. Sur la carrière de d'Aubuisson, cf. la notice d'Arthur Birembaur, « Aubuisson de Voisins, Jean-François d' ", in Dictionary of Scientific Biography, éd. Charles Coulston GILLIsPIE, New York, Charles Scribner, 1970, vol. I, p. 328.

33. Sur l'école de Sorèze, on se reportera à l'ouvrage de Jacques Fabre de Massaguel, L'École de Sorèze de 1758 au 19 fructidor an IV (5 septembre 1796), Issoudun, 1958. Sur l'enseignement dans ces écoles, cf. Robert LEMoINe, " L'enseignement scientifique dans les collèges bénédictins ", in René TATON, éd., Enseignement et diffusion des sciences en France au Xvilf siecle, Paris, Hermann, 1964, p. 101-123.

34. D'après le dépouillement des années 1779-1786 des Exercices publics des élèves de l'École royale militaire de Sorèze tenue par les religieux Bénédictins de la Congrégation de Saint-Maur, Carcassonne. Ces Exercices ont été consultés à la Bibliothèque municipale de Castres (fonds Huillet).

35. Sur la période suivante, cf. le dossier « D'Aubuisson de Voisins, Jean-François » aux Archives de la Guerre, Château de Vincennes, Paris. Sur la nécessité, pour l'artilleur encyclopédiste, d'étudier la minéralogie, cf. la préface à l'ouvrage de LomBarD : Traité du mouvement des projectiles, appliqué aux bouches à feu, Paris, an V, cité in P. CharbonNier, Essais sur l'histoire de la balistique, Paris, 1928, p. 156.

36. Cf. le certificat du prince de Condé (Überlingen, $1^{\text {er }}$ oct. 1797), aux Archives de la Guerre, Paris.

37. Marquis d'Ecquevilly, Campagnes du corps sous les ordres de son altesse sérénissime Mgr le prince de Condé, Paris, 1818, p. 230-231. La marche-route du Quartier général et de la colonne partie d'Überlingen le 8 octobre indique un passage à Freyberg le 25 novembre 1797. 
les rapports $"{ }^{38}$. C'est sous l'influence de Charpentier, conseiller des mines, ancien professeur de mathématiques, d'ascendance française, que d'Aubuisson reconnaîtra des années plus tard être resté deux ans à Freiberg " sans vouloir même suivre les cours de minéralogie de ce professeur $[=$ Werner $] "{ }^{39}$. Et, de fait, d'Aubuisson ne suivra les cours de Werner qu'en 1800 et 1801 , soit vers la fin de son séjour à Freiberg.

Charpentier fils se souviendra, en 1823, de "la bienveillance que M. d'Aubuisson [lui] a témoignée dès [son] enfance, et [de] l'intérêt qu'il a conservé pour [sa] famille ${ }^{40}$.

Accueilli par Charpentier et Werner, d'Aubuisson s'est vu proposer un échange de savoirs : mathématiques françaises contre minéralogie allemande :

« Nous sommes ici [...] mineurs expérimentés, assez bons géologues, mais mathématiciens peu habiles; en France, c'est précisément le contraire. Demeurez à Freiberg; vous nous enseignerez l'algèbre transcendante que nous savons mal, vous étudierez ce que nous savons mieux que vous, et quand votre patrie aura cessé de vous proscrire, vous lui rapporterez des connaissances qui seront utiles à elle et à vous ${ }^{41}$.

C'est ainsi « qu'un Français a pu employer les moments bruyants de la Révolution à recueillir des matériaux précieux pour éclairer les travaux utiles et paisibles du mineur " ${ }^{42}$.

À Freiberg, d'Aubuisson jouit donc d'un double statut : élève en minéralogie, professeur privé en mathématiques, il fait "échange d'études, écoutant et donnant des leçons " ${ }^{43}$. C'est précisément ce double statut qui rend possible, quelques mois plus tard, sa rencontre avec Hardenberg.

38. Annales de chimie, t. 69, p. 237 (souligné par nous). Cf. le témoignage concordant de Hardenberg, in HKA, IV, p. 314.

39. Ibid., p. 238.

40. Jean de Charpentner, Essai sur la constitution géognostique des Pyrénées, Paris, 1823, préface, p. xij, note.

41. De Panat, Éloge de M. d'Aubuisson de Voisins, membre de l'Académie des Jeux floraux, lu en séance publique, le 15 janvier 1843, p. 4-5. Ce texte peut être consulté à la Bibliothèque municipale de Toulouse, cote : LmC 5542.

42. Cette citation, extraite d'une lettre versée au dossier $\mathrm{F}^{14} \cdot 2712^{2}$ des Archives nationales, Paris, porte : Le Conseil des mines au Citoyen Daubuisson à Freiberg en Saxe (26 prairial an $\mathrm{X}$ ). Il s'agit d'une réponse à la lettre de Daubuisson datée du 12 ventôse an $X$. L'insistance de d'Aubuisson sur ce point est particulièrement nette : ne pouvant plus être utile au pays par la voie des armes, il s'agit d'être utile par la voie du savoir, de « faire connaître à la France les détails d'un art utile tels qu'ils sont pratiqués dans un pays où cet art est porté à sa perfection ", afin de " tirer le parti le plus avantageux des richesses minérales du territoire français » (lettres des 16 thermidor an VII et 27 nivôse an XI). En ce sens, ses études en minéralogie allemande l'ont peut-être "mis à même de coopérer à conserver une branche importante d'industrie à la France » (lettre du 26 sept. 1814, cf. Archives de la Guerre, Paris).

43. M. de BOuCHEPORN, " Notice nécrologique sur M. d'Aubuisson de Voisins ", Annales des mines, 1847 , p. 672 . 
Une lettre de ce dernier en porte indirectement témoignage. Datée du $1^{\text {er }}$ septembre 1798, postée de Freiberg et adressée à son père, elle fait état d'une quadruple dette (en Reichsthaler) envers Lampadius, Lempe, d'Aubuisson et Werner ${ }^{44}$ :

« À présent il dépend de toi de m'envoyer l'argent pour Lampadius, Lempe et d'Aubuisson. Avec Werner, cela peut attendre.

\begin{tabular}{lr} 
Lampadius & $100 \mathrm{rch}$. \\
Lempe & $20 \mathrm{rch}$. \\
D'Aubuisson & $18 \mathrm{rch}$. \\
\cline { 2 - 2 } & $138 \mathrm{rch}$.
\end{tabular}

Je dois jusqu'à présent 12 semaines à d'Aubuisson. Je ne puis lui soustraire les 6 semaines où j'étais absent, car il n'y prétend même pas " ${ }^{45}$.

Notons, au passage, l'énorme disproportion de la dette entre Lampadius et Lempe (et d'Aubuisson), entre la chimie et les mathématiques. Selon cette lettre, les cours que d'Aubuisson donna en privé à Hardenberg remonteraient au début du mois de juin 1798 (dette de 12 semaines). Il convient toutefois de décompter les six semaines où Hardenberg fut absent. Cette longue absence est, pour l'essentiel, due à la cure qu'il suivit à Teplitz, du 15 juillet à la mi-août 1798 . C'est de cette période que datent les Teplitzer Fragmente, où le nom de d'Aubuisson apparait une seconde fois, dans un contexte quelque peu différent ${ }^{46}$.

Un peu plus bas dans cette lettre-témoignage, Hardenberg procède à une critique sans aménité de la pédagogie de Lempe et, dans une mise en parallèle stricte, oppose Lempe à d'Aubuisson : « De lui [ = d'Aubuisson] j'apprends vraiment les mathématiques [...] Je n'apprends rien chez lui $[=$ Lempe $] \gg{ }^{47}$. Comment entendre une opposition aussi nette?

44. Soit un professeur de chimie, un professeur de minéralogie et deux professeurs de mathématiques (l'un public, l'autre privé).

45. HKA, IV, p. 259.

46. HKA, II, p. 563 : " L'explication chimique de d'Aubuisson concernant le calcul des lettres. Ces signes ne s'incluent pas comme des nombres, mais on voit encore en chaque composition les éléments, leurs rapports et la méthode de composition. Ils s'associent, mais ils ne se confondent pas " (cette dernière phrase est en français dans le texte). Sur les deux types d'opération (et donc d'écriture) ici présentés, on voudra bien se reporter à notre conclusion. Le rapprochement avec la notation chimique se retrouve, par exemple, dans le livre de Silvestre-François LACRorx, Essai sur l'enseignement en général et sur celui des mathématiques en particulier, Paris, an XIV-1805, p. 242, 246, 250.

47. HKA, IV, p. 259. 


\section{I. - LES ENJEUX D'UNE RENCONIRE : MATHEMATIQUES ET GEOLOGIE}

\section{Mathématiques.}

La rencontre entre Hardenberg et d'Aubuisson peut être placée sous le signe de l'opposition entre la pureté de la mathesis et l'impureté de gaia. Vu les préventions de Charpentier contre Werner, il n'est pas exagéré de prétendre que le contact eut lieu sous les auspices de Charpentier. À l'époque, d'Aubuisson sortait d'une formation à prédominance scientifique et technique, où la mathématique l'emportait. Fait plutôt rare à l'époque chez un jeune homme de vingt-neuf ans, il maîtrisait le « calcul des infinis ". Bref, il détenait un savoir qui privilégiait le pur et la pureté. Il ne pouvait avoir de la nature qu'une vue épurée : « Le vague et le peu de précision qui régnaient dans les descriptions et les déterminations ne pouvaient que rebuter une personne élevée dans l'étude des sciences exactes $"{ }^{48}$. Or, la nature, et tout particulièrement la terre, est ce qui résiste le plus longtemps à l'épuration. La matière est la limite de la fonction, la terre est ce qui reste ${ }^{48 \text { bis }}$. La pureté en minéralogie et géologie est rare et minoritaire. Même une fois surmontées ces réticences et ces préventions (en 1800-1801), d'Aubuisson fait la part du pur et de l'impur au sein du discours wernérien. Chassant, traquant l'impureté au sein du discours minéralogique :

« Après avoir entendu pour la première fois les leçons de Werner sur la géognosie, j'en éloignai tout ce qui me paraissait vague, et je me fis, sur les divers points de la science, des idées où tout était net, exact et précis; mais lorsque j'allai ensuite observer la nature, je n'y trouvai plus rien qui répondît à ces idées $"{ }^{49}$.

D'Aubuisson apprendra à Freiberg et en d'autres lieux à se déprendre du geste cartésien, à ne point écarter l'impur.

Grâce à son ami Thielmann, Hardenberg est accueilli dès le début de l'année 1798 dans la famille Charpentier et suit les cours de mathéma-

48. Annales de chimie, Paris, t. 69, p. 238.

48 bis. Cf. notre communication au séminaire de l'Institut international de géopoétique sous la dir. de Kenneth White : " La poétique de la terre chez Novalis ", Université de ParisSorbonne, avril 1992, à paraittre dans les Cahiers de géopoétique, Genève, Zoé, 3, 1992.

49. Jean-François d'Aubuisson de Voisins, Traité de géognosie, Paris, 1828, nouv. éd. revue et corrigée, p. 373. Goethe utilisera, non sans quelque réticence, ce traité. Cf. Goethes Werke, Hambourg, C. Wegner, 1967, Bd XII, p. 402. 
tiques de Lempe, ancien élève de Charpentier précisément. À Leipzig, Hardenberg avait sans doute suivi les cours de Hindenburg, père de l'école combinatoire allemande ${ }^{50}$ : autant dire qu'il était familiarisé avec le statut mathématique des combinaisons et des opérations combinatoires $^{51}$. C'est pourquoi, dès février 1798 , Hardenberg se plaint qu'on le traite, à Freiberg, comme s'il en était encore à l'alphabet ${ }^{52}$.

Les cours de l'année 1798-1799 sont consacrés à l'arithmétique, la géométrie, la trigonométrie plane et sphérique, à la physique théorique et expérimentale, à la mathématique appliquée, à la mécanique appliquée aux mines.

Face à une certaine pédagogie allemande, Hardenberg a d'ailleurs les mêmes réactions que d'Aubuisson élève. Tous deux faisaient preuve d'une certaine maturité (celui-ci avait, à l'époque, vingt-neuf ans, celui-là, vingt-six ans) face aux jeunes élèves admis à la Bergakademie. De plus, tous deux avaient déjà derrière eux une formation mathématique ${ }^{33}$. Mais Hardenberg procède, dans la lettre citée, à une critique sans aménité de la pédagogie de Lempe : "Il lit de façon extrêmement désagréable, parcourt ce qu'il peut et est satisfait quand il peut dire : j'ai fait mon cours. Werner et Lampadius sont meilleurs en ce domaine $"{ }^{54}$. Ce que Hardenberg reproche, c'est donc une froide lecture au lieu d'une matière exposée d'abondance, avec enchaînement et série de gradations dans les idées qui pénètrent l'auditeur jusqu'à l'enthousiasme. En outre, Lempe ne se donne pas la peine de pousser plus loin, de pousser plus avant ses auditeurs ${ }^{35}$.

Mais il y a plus décisif encore : n'oublions pas en effet que Lempe, successeur de Johann Wilhelm von Charpentier à la chaire de mathématiques et physique, est avant tout un mécanicien. Éditeur du Magazin für die Bergbaukunde ${ }^{36}$, il est l'auteur d'un ouvrage de référence en mécanique ${ }^{57}$.

50. Cf. M. Dyck, op. cit. supra n. 4, p. 31 ; Moritz CANTOR, Vorlesungen über Geschichte der Mathematik, vierter Band, New York/Stuttgart, Teubner, 1965, p. 4-5, 201-221.

51. Sur le statut des permutations, of. G. Th. Gullaud, "Qu'est-ce qu'une permutation?", Mathématiques et sciences humaines, 42, 1973, p. 5-16; Emest Coumrt, Mersenne, Frenicle et l'élaboration de l'analyse combinatoire dans la première moitie du xhf siècle, thèse de doctorat, Paris, 1968 ; ID., " Des permutations aux xvic et xvir siècles ", in Permutations, Actes du colloque sur les permutations, Paris, Université René-Descartes, 10-13 juil. 1972, publié par la Société mathématique de France, Paris/La Haye, Mouton, Paris/ Bruxelles/Montréal, Gauthier-Villars, 1974.

52. HKA, IV, p. 251.

53. Pour Hardenberg, cf. M. Dyck, op. cit. supra n. 4, p. 31.

54. HKA, IV, p. 260.

55. HKA, IV, p. 259-260.

56. Johann Friedrich LEMPE, Hrsg., Magazin fur die Bergbaukunde, Dresde, 1785-1799.

57. ID., Lehrbegriff der Maschinenlehre, mit Rilcksicht auf den Bergbau, Leipzig, 17951797,2 tomes parus. 
En tête de son ouvrage figure une longue bibliographie commentée ${ }^{58}$ où les mécaniciens français suivants sont cités : d'Alembert (Traité de dynamique, 1743, 1752; Traité de l'équilibre et du mouvement des fluides, 1744), Bossut (Traité élémentaire de mécanique, 1763, 1775; Traité élémentaire d'hydrodynamique, 1775, 1777), Buat (Principes d'hydraulique, $1779,1786)^{59}$, Lagrange (Mécanique analytique, 1788), Prony (Nouvelle architecture hydraulique, 1790).

L'exposé des principes de la mécanique de Lempe permet de mieux comprendre tout ce qui l'oppose à Hardenberg : Lempe remonte des contraintes techniques (technische Maschinenkunde) à la mathématique supérieure (mathematische Maschinenkunde), de l'application aux principes, du particulier au général ${ }^{60}$. La présentation de cas réels, la confrontation avec les difficultés mécaniques, telle est la première école du mécanicien. L'étude des contraintes et des circonstances doit précéder, et non suivre, l'introduction de la mathématique supérieure. Hardenberg procède de façon inverse : il s'installe d'emblée dans la généralité, dans l'abstraction, pour comprendre les cas singuliers. Ainsi la saisie du singulier vient toujours en fin de parcours.

En dépit de ses critiques à l'encontre de Lempe, Hardenberg n'en méprise pas pour autant la mécanique, bien au contraire. Il s'agit de lui assigner une place dans le système encyclopédique ${ }^{61}$. Et cette assignation ne peut avoir lieu qu'à la condition expresse de prendre en compte la partition - et le jeu réglé - entre le pur et l'appliqué. Pour mieux situer la mécanique, Hardenberg se propose, par un détour, d'acquérir de la hauteur. Ce détour n'est autre que le passage par le calcul (différentiel et intégral) qui permettra de se retourner ensuite, avec un maximum de compréhension, vers la mécanique.

Qu'est-ce alors que la vraie mathématique, pour Hardenberg? Celle qui s'est dégagée de toute application préalable, pour mieux s'appliquer ensuite $^{62}$; celle qui est d'autant plus applicable, qu'elle est moins appliquée d'abord; celle qui recherche la pureté de la mathesis pour mieux comprendre l'impur.

58. Ibid., p. 11-58.

59. Lempe traduira cet ouvrage de Buat en allemand, en 1795. Sur la mécanique de Buat, cf. René Dugas, Histoire de la mécanique, Neuchâtel/Paris, Dunod, 1950, p. 303-305.

60. Le troisième volume du Traité de Lempe, qui devait être consacré à la mécanique mathematique, n'a jamais vu le jour (Lempe est décédé en 1801).

61. Sur la fonction de la mécanique dans le romantisme, cf. Bernhild Bore, L'Homme et ses simulacres, Paris, José Corti, 1979, en part. p. 289-294.

62. Cf. Michel SERRES, "Les anamnèses mathématiques", in Hermès I. La communication, Paris, Ed. de Minuit, 1968, p. 50 sq. et 101 : « Le jugement récurrent [est un] jugement d'application. Pour nous, la géométrie de Thalès est une métrique de maitre-maçon, celle de Desargues est d'un expert en taille de pierres, en trompes et escaliers, la géométrie cartésienne est celle d'un ingénieur, celle de Monge est d'architecte à son lavis (elle fut dite descriptive) [...] " (souligné par nous). 
Les études de Hardenberg ${ }^{63}$ ont été extrêmement précises et puisées, en particulier, aux sources françaises de deux manuels de mathématiques : les Traités de calcul différentiel et de calcul intégral de Bossut ${ }^{64}$ et le Cours de mathématiques à l'usage de la marine et de l'artillerie de Bézout ${ }^{65}$.

Coïncidence troublante puisque, nous l'avons vu, d'Aubuisson luimême avait étudié à Sorèze les mathématiques dans les manuels de Bossut (mécanique : statique + dynamique) et Bézout (arithmétique, géométrie, algèbre, calcul des infinis) ${ }^{66}$.

C'est pourquoi il n'est pas invraisemblable de supposer que d'Aubuisson avait proposé ces manuels à la sagacité studieuse de Hardenberg. Mais, d'autre part, Bossut mécanicien et hydrodynamicien n'était pas inconnu de Lempe. De même, il avait inscrit à son cours de mathématiques la trigonométrie sphérique et pouvait avoir connaissance des manuels de Bézout.

Pourquoi le calcul et la trigonométrie sphérique? L'un comme l'autre valent comme introduction à d'autres régions, à d'autres domaines de l'encyclopédie ${ }^{67}$. Sortes de passages obligés si l'on veut manifester ce qui est au fondement. Et, précisément, par cette étude Hardenberg se déprend des séductions de l'art de l'ingénieur et opère un retour de l'application au fondement - de la mécanique au calcul, de l'astronomie à la trigonométrie sphérique.

63. Cf. Jean Dhomвres, « French Mathematical Textbooks from Bézout to Cauchy », Historia Scientiarum, 28, 1985, p. 91-137; " Mathématisation et communauté scientifique française ", Archives internationales d'histoire des sciences, vol. 36, 117, 1986, p. 249-287; Nicole et Jean Dhombres, Naissance d'un pouvoir : sciences et savants en France (1793-1824), Paris, Payot, 1989.

64. Charles Bossur, Traités de calcul différentiel et de calcul intégral, Paris, an VI, 1798, tome $\mathrm{I}^{\text {er }}$. Cf. HKA, III, p. 115-119. Dans ces traités, Hardenberg étudiera les cent premières pages environ, soit les chapitres consacrés au calcul aux différences finies (calcul différentiel aux différences finies, calcul intégral aux différences finies).

65. Étienne BEzour, Cours de mathématiques à l'usage de la marine et de l'artillerie (Edition originale, revue et augmentée par Peyrard, renfermant toutes les connaissances mathématiques nécessaires pour l'admission à l'École polytechnique), Paris, an VI, 1798 - seconde partie - Éléments de géométrie, trigonométrie rectiligne et trigonométrie sphérique. Cf. HKA, III, p. 167 et HKA, V, p. 59-60 (résumé). L'origine du document a été établie par nos soins, in Daniel Lancereau, Novalis, Bézout et Bossut, Les manuels de mathématiques dans la tradition française de l'Encyclopédie, à paraitre.

66. Sur Charles Bossut et Étienne Bézout, on consultera : C. Stewart Gillmor, « Bossut, Charles », in Dictionary of Scientific Biography, op. cit. supra n. 32, vol. II, p. 334-335 et Judith V. Grabiner, « Bézout, Étienne », in ibid., p. 111-114.

Sur le rôle considérable des examinateurs et, en retour, des manuels en vigueur, cf. Roger HAHN, « L'enseignement scientifique aux écoles militaires et d'artillerie », in René TATON, op. cit. supra n. 33, p. 519, 529, 533; Denis I. DuveEN, Roger HAHN, « Laplace's Succession to Bézout's Post of examinateur des élèves de l'artillerie $»_{i}$,Isis, 1957, p. 416-427.

67. Lorsque l'universitas fait défaut, il ne reste plus qu'à corréler les régions par un système de correspondances. Sur le statut des correspondances, cf. J.-L. ViellLARD-Baron, « Microcosme et macrocosme chez Novalis », Les études philosophiques, 2, 1983, p. 195-208. 
Que retire-t-il de ces études? Du calcul, il retire un procédé d'écriture qui consiste à passer du local au global, à trouver ou à frayer un chemin (ou plusieurs) qui ponte les variétés locales ${ }^{68}$. En ce sens, l'écriture est un déploiement dont « le domaine n'est pas circonscrit à l'avance, mais résulte plutôt de la succession infinie des opérations locales $"{ }^{69}$. Or cette idée de déploiement (et de déploiement universel) est « un élément très important de cette constellation d'idées qui accompagnent l'apparition du calcul différentiel ${ }^{70}$.

Ce déploiement a pour autre nom, chez Hardenberg : empirisme actif (tätiger Empirismus). C'est la Tätigkeit ${ }^{71}$ qui nous arrache à l'empirisme, au tourbillon local de l'empirisme (Strudel der Empirie) ${ }^{72}$. Mais inversement : il est possible de condenser, de plier une figure globale en un point (singularité). Ces deux procédés réciproques (déploiement/repliement) trouvent une application aussi bien en mathématiques ${ }^{73}$ qu'en poé$\operatorname{sie}^{74}$.

Parallèlement, la méthode de triangulation sur sphère (trigonométrie sphérique), d'utilisation canonique en astronomie, assure la maîtrise de la position des astres : elle permet le calcul des longitudes et des latitudes, des ascensions droites et des déclinaisons des astres. À sa façon, elle permet une double opération : réduire la part du hasard, de l'arbitraire, de l'éparpillement; étendre le déterminisme de la connaissance. La méthode permet de mettre de l'ordre, c'est-à-dire du savoir et de la prédiction,

68. Cf. Jean-Francois MARQuET, "Chaos et culture dans les philosophies du romantisme allemand ", Les études philosophiques, 1, 1983, p. 53-68.

69. Albert Laumann, Essai sur l'unité des mathématiques, et divers écrits, Paris, Union générale d'éditions, 1977, p. 33.

70. René Thом, Paraboles et catastrophes, Paris, Flammarion, 1983, p. 28.

71. Sur l'arrière-plan philosophique de la Tätigkeit, cf. J.G. FichIE, Grundlage der gesammten Wissenschaftslehre, in J.G. FICHTE, Gesamtausgabe, Stuttgart/Bad Cannstatt, 1965, I, 2, p. 249-451 ; Grundriss des Eigenthulmlichen der Wissenschaftslehre, in ibid., 1966, I, 3, p. 137-208; Grundlage des Naturrechts nach Prinzipien der Wissenschaftslehre, in ibid., I, 3, p. $311-460$; J. G. Fichre, CEuvres choisies de philosophie première, trad. franç. d'Alexis Philonenko, Paris, Vrin, 1980.

Sur l'arrière-plan religieux, cf. Max Weber, L'Éthique protestante et l'esprit du capitalisme, trad. franç. de Jacques ChAVy, Paris, Plon, 1981.

72. HKA, IV, p. 251.

73. Cf. R. THом, Modeles mathematiques de la morphogenèse, Paris, C. Bourgois, 1981, p. 116, 162 : " ... le mathématicien dispose, pour aller du local au global, d'une notion sûre : l'analyticité [...] Pour passer du global au local, [il] dispose d'une autre notion, celle de singularité [...]. C'est par l'emploi alterné [...] de ces deux techniques [...] qu'on peut espérer aboutir à une synthèse de situations globales complexes. "

74. Pour Hardenberg, le roman se conçoit comme déploiement d'un germe, qui se trouve être l'analogue d'une section rimante. Nous avons abordé l'isomorphisme entre les ta mathemata et les ta littera dans notre communication : « Poésie, philosophie et science chez Novalis " (décembre 1990) au séminaire du C.R.D.H.M., à paraître in Les études philosophiques, janv.-mars 1993. 
dans une nature foncièrement météorique ${ }^{75}$. Elle permet d'encadrer l'aléa des circonstances.

C'est que la trigonométrie permet, en retrait de la météorologie et de l'astronomie, de recueillir la donne des éléments : soleil et météores (nuages, vents, grêle, trombes d'eau, giboulées, etc.). C'est elle qui organise l'espace du recueil ${ }^{76}$. À l'intérieur de cet espace par elle déployé, Hardenberg tentera d'optimiser les opérations nécessaires à l'obtention du sel - graduation (Gradierung) et cuite (Siedung).

Dès lors, qu'il s'agisse d'extraire le poème de la langue, ou le sel de la mine, tout est pour Hardenberg occasion de production. Qu'elles relèvent du calcul ou de la trigonométrie, ses études pratiquent une traversée de l'encyclopédie qui répond à un double souci : maîtriser l'événement pur, la circonstance, établir en quelque sorte un savoir des météores; étendre l'empire de la substance à la fonction, établir de proche en proche des passages, des chemins du local au global.

Le déploiement de la Tätigkeit est aussi un exercice d'écriture, autrement dit une manière d'opérer qui manifeste : la différence entre le calcul direct (différentiel) et le calcul indirect (intégral); la différence entre la décomposition (la différenciation décompose les grandeurs) et la recomposition (l'intégration les rétablit); l'importance de la méthode d'exhaustion des anciens géomètres (dans la tradition eudoxienne et archimédienne); la notion d'opération et d'opération réversible (possibilité d'inverser l'ordre des opérations).

Chez Hardenberg, l'inversion est de méthode : « Tout comme Copernic, les bons chercheurs le font - les médecins, et les observateurs, et les penseurs - : ils inversent [drehen ... um] les données et la méthode, pour voir si cela ne va pas mieux ainsi $"{ }^{77}$. Mais elle-même n'est qu'un cas de figure de la variation des points de vue : inverser n'est qu'une façon, fort singulière et fort restreinte, de varier. À nouveau, la question rebondit : pourquoi varier? La variation est de rigueur si l'on veut épuiser les possibles. Autrement dit : il ne suffit pas d'inverser, il faut varier; mais il ne suffit pas non plus de varier, il faut varier infiniment, sous peine d'incomplétude. C'est ainsi que l'inversion pourra mener à l'exhaustion, via la variation.

Hardenberg ne se contente pas de nommer l'inversion : cette figure est elle-même soumise à variation : elle est l'aller et le retour, l'avancée et le recul, la progression et la régression. Elle s'applique en tous domaines :

75. Jean Leveque, Le Fragment I, Paris, Osiris, 1989; ID., Récit, désir, mathématique, Paris, Osiris, 1989; ID., Le Jeu et le temps, Paris, Osiris, 1990.

76. Cf. l'utilisation par Hardenberg de l'ouvrage de Joseph Jérôme LaLANDE, Astronomisches Handbuch oder die Stermkunst, Leipzig, 1775, in HKA, III, p. 170-171.

77. HKA, III, p. 355. 
philosophie, logique, mathématiques, sciences, etc. Elle met en jeu les oppositions suivantes : l'analyse et la synthèse, l'intuition et le concept, l'abstrait et le sensible, les choses et les abstractions, l'idéalisme et le réalisme, etc. D'où l'absurdité de ne parler que d'un seul moment; de concevoir, d'imaginer même un chemin qui puisse être sans retour. Le trop célèbre chemin vers l'intérieur (Der geheimnisvolle Weg nach Innen $)^{78}$ n'est rien s'il est sans retour. Que serait, dans la problématique de Hardenberg, qui est une problématique feuilletée, un chemin, une voie à sens unique? Précisément : un chemin parcouru demande à l'être encore - dans l'autre sens, en attente d'une variation plus large, plus ample. Tout chemin est susceptible d'être investi à partir de tous les points de vue.

"Les deux opérations sont idéalistes. Celui qui les a toutes deux en son pouvoir est l'idéaliste magique $"{ }^{79}$. On comprend mieux dès lors l'expression : "idéalisme magique », chère à Hardenberg ${ }^{80}$. L'idéaliste magique est celui qui tient en main les opérations et les opérateurs, et les maîtrise d'autant mieux qu'ils sont réversibles. Seul celui qui tient en son pouvoir ces opérations (mais nous savons qu'à travers l'inversion, c'est la variation, puis l'exhaustion qui sont en jeu) peut être déclaré idéaliste magique. C'est pourquoi, des Fichte-Studien au calcul infinitésimal, l'ordo inversus - entendu en langue universelle - est bien le maitre-mot de l'idéalisme magique.

\section{Géologie}

Après avoir examiné la rencontre entre Hardenberg et d'Aubuisson au niveau des mathématiques, il convient d'aller au-delà. La rencontre se poursuit, mais sur un autre terrain : celui de la minéralogie.

Une comparaison s'impose ici entre l'élève minéralogiste Hardenberg et l'élève d'Aubuisson. À cette occasion, le rapprochement des textes va nous être d'un grand secours : pour Hardenberg, nous prendrons en compte les Werner-Studien ${ }^{81}$ et Das Allgemeine Brouillon ${ }^{82}$; pour d'Aubuisson, les différents mémoires, publiés de 1801 à $1809^{83}$ qui

78. HKA, II, p. 418 sq. et III, p. 434.

79. HKA, III, p. 301 et aussi p. 58.

80. Sur l'idéalisme magique, cf. HKA, II, p. 546, 605 ; HKA, III, p. $315,385,430$, etc. Nous n'abordons pas ici le problème de la " mathématique mystique " chez Hardenberg. II est traité dans l'ouvrage de Dietrich MAHNKE, Unendliche Sphäre und Allmittelpunkt, Halle, 1937, rééd. Stuttgart/Bad Cannstatt, 1966, p. 1-5.

81. HKA, III, p. $135-161$.

82. HKA, III, p. 242-478.

83. De 1801 à 1809 , d'Aubuisson a fait paraitre ses exposés sur la minéralogie allemande et la méthode wernérienne dans trois revues : le Journal des mines, Paris, vendémiaire an $\mathrm{X}$, p. 63-90; ventôse an X, p. 517-520; germinal an X, p. 23-57; floréal an X, p. 121-152; le Journal de physique, de chimie et d'histoire naturelle, Paris, ventôse an X, p. 205-240; floréal 
constituent pour ainsi dire une défense et illustration de la méthode wernérienne.

Hardenberg s'occupe à Freiberg de travaux pratiques et techniques; il recueille par écrit les cours qu'il suit, les lectures qu'il peut faire en tous domaines. Son parcours, nous l'avons dit, est une véritable traversée de l'encyclopédie, laquelle est conçue comme un exercice de la pensée : "Pour ce qui est de la classification et de la définition, etc., je veux m'exercer [üben] sur le système de Werner et sur les sciences $"{ }^{84}$.

Le système de classification de Werner sera un des lieux d'une explication avec son maître, une occasion majeure de s'exercer ${ }^{85}$. C'est que le grand esprit systématique de Werner (Werners grosser systematischer Geist $^{86}$ a su créer une langue minéralogique, soit un alphabet et une syntaxe - une sorte de système " convoluté » (ein systematisches Convolut) ${ }^{87}$ qui présente toutefois des lacunes (Lücken).

Qu'en est-il de cet exercice? Il ne doit pas être considéré de façon isolée ; il est inséparable d'autres tentatives, d'autres lectures, d'autres applications; c'est, pour ainsi dire, un échantillon d'exercice. Qu'en est-il de l'objet même de cet exercice? L'ouvrage de Werner : Von den äusserlichen Kennzeichen der Fossilien est daté de 1774. C'est donc une œuvre de jeunesse dont l'auteur a alors vingt-cinq ans. En 1798, il disposait d'un recul de vingt-quatre ans par rapport à cette cuvre. D'autre part, il publie peu, car il procède à des changements continuels dans son système. De cette façon, par avancées successives, il essaie d'épouser au mieux son objet. C'est donc avec un ouvrage tout à fait particulier que Hardenberg se propose d'engager le dialogue.

Dans son approche de la minéralogie, Hardenberg pose d'emblée le problème de la puissance de la systématique. Il n'est, pour lui, pas d'équipuissance des systèmes : certains obéissent à une logique faible, d'autres à une logique forte. Le système oryctognosique de Werner est de faible systématisation; cette faiblesse vient de ce que le système tout entier est soumis à l'utile, au prosaïque (Die nützliche prosaische Oryktognosie... ein schwacher Versuch der mineralogischen Systematik) ${ }^{88}$.

Ce que Hardenberg ne dit pas, et que d'Aubuisson dira (et dira d'autant mieux qu'il possède un recul culturel que Hardenberg ne possé-

an $X$, p. 333-344 ; prairial an X, p. 414-420 ; messidor an $X$, p. 10-21 ; frimaire an $X$, p. 448475 ; les Annales de chimie, Paris, t. 57,1806 , p. $273-316$; t. 62, 1807, p. 302-322; t. 69, 1809 , p. $155-188$ et $225-248$.

84. HKA, III, p. 363.

85. Heinz Dieter ScHmID, Friedrich von Hardenberg (Novalis) und Abraham Gottlob Werner, Tübingen, 1951, Diss. Masch.

86. HKA, IV, p. 298.

87. HKA, III, p. 367.

88. HKA, III, p. 358. 
dait pas encore), c'est le poids des contraintes dues à l'auditoire de Werner et à la finalité de l'enseignement dispensé. Werner, en effet, n'officie pas devant un public savant, mais devant de jeunes élèves de la Bergakademie. Ceux-ci sortent des écoles primaires du pays et leurs connaissances sont " très bornées " ${ }^{89}$.

D'Aubuisson reconnaît un "coloris scolastique et presque trivial " au mode d'enseigner de Werner; mais pour préciser aussitôt qu'un tel état de choses tient à son auditoire : une fois " sorti de son auditoire, il n'a plus rien qui rappelle le ton scolastique ". Imaginons qu'il ait, par extraordinaire, enseigné à Paris, « son mode didactique aurait été alors tout autre de ce qu'il est, et de ce qu'il devait être à l'école de Freiberg " ${ }^{90}$.

La finalité de l'enseignement doit aussi être prise en compte : ces jeunes élèves sont destinés aux travaux des mines, et la majeure partie d'entre eux occuperont des emplois " peu relevés " ${ }^{91}$. C'est pourquoi la pédagogie de Werner consistera à faire passer, sous les yeux des élèves et entre leurs mains, des suites, des échantillons, des variétés de minéraux ${ }^{92}$. On montre les minéraux en grand nombre, ce qui est une exigence au même titre que la variété des suites. On expose ainsi de nombreuses et différentes manières d'être et d'apparaitre.

Il faut bien commencer ici ou là. Mais les descriptions de Werner sont trop individuelles, dira Hardenberg, sa théorie du processus d'observation, trop spéciale (Theorie eines speciellen Beobachtungsprocesses) ${ }^{93}$. Hardenberg ne peut ni ne veut en rester là : il faut aller au plus général, au plus universel. C'est que les fils (Fäden) entremêlés du savoir forment une manière de réseau (Gewebe), c'est-à-dire une table à plusieurs entrées, modèle autrement plus riche que le raisonnement en ligne. Qu'est-ce, précisément, que l'universel, sinon le plus différencié, le plus complexe ? Hardenberg demandera l'application, aux séries oryctognosiques, d'une loi d'élévation pro gradu (Graderhöhungsgesetz) vers le compliqué, le général.

Voilà pour la méthode; venons-en aux applications. À la fin du siècle, la minéralogie se trouve dans une période d'indécision : tous les corps n'ont pas été soumis à l'analyse chimique; de plus, la chimie, bien éloignée de sa perfection, est encore dans l'enfance de l'art. L'occasion est donnée d'éprouver la puissance de la méthode oryctognosique.

Mais qu'est-ce, au juste, que l'oryctognosie? Une manière d'envisager

89. Joumal de physique, de chimie et d'histoire naturelle, floréal an XIII, p. 337.; Annales de chimie, t. 69, p. 231,238 sq.

90. Ibid., p. 232.

91. Ibid., p. 231.

92. Henrich Steffens, Was ich erlebte, vierter Band, Breslau, 1841, p. 207-209.

93. HKA, III, p. 437. 
les minéraux et, conséquemment, l'une des cinq branches de la minéralogie avec la chimie minérale (qui livre le détail de la composition chimique et des parties constituantes), la géognosie (qui étudie les circonstances de leurs gisements), la minéralogie géographique (qui donne les lieux et les pays où on les trouve) et la minéralogie économique (qui dit l'utilité que l'homme en retire, les usages qu'il en fait).

L'oryctognosie échappe, pour l'essentiel, à la clôture du laboratoire. Elle doit être à même de « donner des moyens suffisants, mais simples et faciles dans leur application, pour reconnaître les divers minéraux par le secours immédiat de nos sens ". Ces moyens doivent permettre (au milieu des montagnes, ou à l'intérieur des mines - et ceci est essentiel) de distinguer sur-le-champ les minéraux, en utilisant « les caractères que [les] sens, sans intermède, trouvent dans les minéraux $~^{94}$.

En suivant cette manière de voir, on s'intéressera aux propriétés que les minéraux présentent dans leur état naturel, c'est-à-dire aux caractères extérieurs ${ }^{95}$. Hardenberg s'interrogera sur la définition du signe caractéristique (äusseres Merkmal) ${ }^{96}$; celle-ci est-elle immédiate ou asservie à des opérations minéralogiques (mineralogische Operationen) ${ }^{97}$ telles que : rayer, briser, etc. ?

Quel est le domaine de définition de l'oryctognosie? La classification porte sur les minéraux mécaniquement simples; les mélanges en sont exclus. Mais lorsque les parties mélangées sont si petites qu'elles sont indiscernables à l'œil et que l'ensemble présente une masse homogène, le minéral appartient tout de même au domaine de l'oryctognosie. L'oryctognosie est donc bien une caractéristique, que Werner soumet à tabulation $^{98}$.

Werner - et Hardenberg après lui - se pose la question d'une application possible de la caractéristique, de la symptomatique à la chimie, du passage de l'une à l'autre. Celui-ci est-il possible ? À quelles conditions est-il possible? Est-il toujours possible ${ }^{99}$ ?

Pour Werner, cité par Hardenberg, le passage de l'une à l'autre est possible à la condition de connaitre à l'avance les proportions de la composition; il conçoit donc un passage a posteriori. Werner s'attire la critique de Hardenberg pour une raison que nous connaissons bien désormais : le

94. Journal de physique..., floréal an X, p. 334.

95. Ibid., p. 335 : «Ainsi, par le mot caractères extérieurs, Werner désigne les mêmes caractères que Haüy a nommés caractères physiques (l'électricité exceptée). "

96. HKA, III, p. 358.

97. HKA, III, p. 375.

98. Cf. les huit tableaux dans l'ouvrage de A.G. Werner, Von den ausserlichen Kennzeichen der Fossilien, Leipzig, 1774, p. 86, 128, 206, 207, 226, 256, 264, 282.

99. Cf. Peter KaPITZA, Die Frihromantische Theorie der Mischung, Munich, M. Hueber, 1968. 
primat, la toute-puissance de la méthode. Ce qui vaut dans toutes les régions de l'encyclopédie, vaut aussi en minéralogie : la figure de l'ordo inversus règne ici comme ailleurs. Entre l'aller (Gang) et le retour (Rückgang), Hardenberg inscrit le passage (Übergang), tente de dégager une théorie des passages. Ces passages sont réglés par une certaine mathématicité (Mathematicität) ${ }^{100}$ de l'oryctognosie qui tire, qui extrait l'inconnu du connu.

Pour Hardenberg le passage de la caractéristique à l'essence est possible a priori. Et de qualifier, sur la lancée, la position de Werner de dogmatique. L'idéalisme au contraire, par la puissance de la seule méthode (durch die blosse Methode) ${ }^{101}$, est capable de déterminer les changements de proportions d'après les variations de la caractéristique.

L'application de la symptomatique à l'essence peut être a posteriori, comme le croit Werner en 1774, ou a priori, comme le pense Hardenberg en 1798. C'est que le point de départ est différent pour l'un et pour l'autre. Pour Werner, la composition des minéraux en proportion déterminée et invariante est un cas minoritaire dans la nature; dès lors, la détermination des espèces est soumise à la reconnaissance toujours hasardeuse, toujours relative, des symptômes. Pour Hardenberg, en revanche, ici comme ailleurs, le problème est de méthode ${ }^{102}$.

C'est pourquoi Hardenberg s'en prend à ce qu'il nomme le dogmatisme (Dogmatismus) de Werner. Â ses yeux, la seule méthode, si elle est suffisamment forte, puissante, doit nous permettre de passer du signe au composant, du symptôme à l'élément, de déterminer les modifications de formes et de mélanges, d'établir des lois de correspondances, des règles d'expression ou de détermination réciproque (Wechselbestimmungsregeln). Si la méthode n'en est pas capable, la nature du passage dans l'essence reste méconnue. Il estime que si l'on observe de façon suffisamment suivie et différenciée les modifications extérieures lors de modifications internes, et inversement, l'on aboutira à des règles de détermination réciproque. Trouver la réciproque est bien, là aussi, l'enjeu du discours de la méthode selon Hardenberg.

Les critiques de Hardenberg envers Werner sont-elles fondées? Le sont-elles suffisamment ? Ou s'appliquent-elles seulement à l'ouvrage cité de 1774 ? Les différents mémoires de d'Aubuisson vont nous permettre de mieux situer ces critiques.

Il y a d'abord quelque injustice à juger la méthode de Werner à partir d'une œuvre écrite lorsqu'il avait vingt-cinq ans. Mais ne perdons pas de

100. HKA, III, p. 386.

101. HKA, III, p. 139.

102. Hardenberg établit un passage entre minéralogie, mathématiques et poésie, par l'intermédiaire de la notion de symptôme a priori. Cf. HKA, III, p. $351,386$. 
vue que Hardenberg juge moins qu'il ne s'exerce. D'autre part, on l'a dit, Werner a une répugnance certaine à écrire. Cet ouvrage était sans doute le seul disponible par lequel Hardenberg pouvait aborder le système wernérien. Pour Werner, l'écriture peut commencer son œuvre lorsque des invariants ont été atteints et établis : «Je suis bien loin de regarder l'ordre que j'ai suivi comme parfait; j'y fais des changements toutes les années ${ }^{103}$.

Le bonheur du botaniste, du zoologiste, c'est que, dans ces deux branches, la nature a fait les espèces ${ }^{104}$. Il n'en vă pas de même en minéralogie ${ }^{105}$, car jamais les espèces minérales ne seront distinctes et caractérisées comme les espèces végétales et animales : le vague tient ici à la nature et à la formation des minéraux - c'est le malheur du minéralogiste.

Werner part du principe de composition; c'est le principe le plus simple qui soit. Il l'a proposé dès 1773 , à une époque où la chimie minérale n'existait pas encore. Ce principe s'énonce comme suit : « Tous les minéraux [...] doivent être classés d'après la considération de leur composition " ${ }^{106}$. Si les minéraux sont composés de substances simples, toutes les différences proviennent des différences de composition. La composition fait l'essence du minéral, la forme est un moyen de le reconnaitre. Le signe extérieur fait reconnaittre la substance, la composition exprime son essence.

Il existe deux grandes classes de minéraux : les minéraux à composition invariable et les minéraux à composition variable. Dans la première classe, les éléments se combinent toujours selon des proportions fixes et invariables. S'il y avait autant de combinaisons des principes constituants que d'espèces, on pourrait dire alors, qu'en minéralogie aussi, la nature fait les espèces. Or il y a, et nous n'y pouvons rien, variété et variation au sein des proportions et des rapports : les combinaisons se rencontrent, multiples, en proportions variables.

La seconde classe (les minéraux à composition variable) comporte deux sous-classes : celle où la composition des minéraux varie entre certaines limites (dans ce cas aussi on pourrait dire que la nature a fait les espèces), et celle pour lesquels il n'y a aucune fixité. Dans ce dernier cas, les espèces sont des êtres de convention ${ }^{107}$. C'est lorsque la proportion se fait indéterminée, variable à l'extrême, lorsque le principe de composition

103. Journal de physique..., nivôse an XIII, p. 171.

104. Annales de chimie, t. 57, p. 295.

105. Pour Hardenberg, en revanche, la doctrine de classification botanique et zoologique est beaucoup plus compliquée que la doctrine de classification minéralogique. CF. HKA, III, p. 392.

106. Annales de chimie, t. 57, p. 283-284.

107. Ibid., t. 69, p. 178, 183-184. 
n'est plus à même de se prononcer, qu'il est nécessaire de recourir à la convention, aux règles de convenance, afin de suppléer au principe de composition.

Pour faire face à l'arbitraire, deux stratégies de suppléance sont à notre disposition : mise en place de règles de substitution, d'une part; multiplication des points de vue, d'autre part.

De 1773 à 1798, l'évolution de Werner est impressionnante : parti d'un principe sans restriction, il se déprend peu à peu de ces règles trop strictes, trop infidèles à la nature, pour leur substituer, à chaque fois, des règles plus lâches :

"C'est précisément pour être, le plus qu'il lui serait possible, d'accord avec la nature [...], qu'il a successivement secoué le joug de la plupart des principes qu'il avait lui-même posés autrefois. En 1773 [...], il établissait, de la manière la plus tranchée et sans restriction, un principe pour la formation des espèces [...]; en 1780 [...], il formait le genre d'après la partie constituante qui se trouvait dans un minéral en plus grande quantité : quelques années après, [...], il se départit de cette règle, et, pour mieux faire, il lui en substitua une autre bien moins précise et qui prêtait à l'arbitraire. En 1798, je l'ai vu assez embarrassé sur la manière dont on devait suppléer et interpréter son propre principe spécifique : $j$ 'ai ouĩ dire que son incertitude a encore augmenté depuis ; de sorte que plus il observe, et plus ses principes de classification deviennent lâches ${ }^{108}$.

Plus Werner observe, plus il est amené à substituer à des principes fermes des principes plus lâches; il leur accorde, à chaque remaniement, un degré supplémentaire de liberté, mais une liberté qui assure une fidélité plus grande. Plus il observe, plus il se déplace, plus il substitue, plus il différencie. C'est parce qu'il y a convenance et convention que Werner peut bousculer les règles qu'il a lui-même établies : ses déplacements incessants sont autorisés, réglés par le principe de convenance. Si celui-ci venait à disparaître, le voyage s'arrêterait, les espèces seraient fixées, l'impression du livre pourrait commencer. Il n'y a donc pas de dogmatisme chez Werner, si ce n'est le dogmatisme de la jeunesse. Ce qui peut faire comprendre la position de Hardenberg. Mais Werner met en place, année après année, des règles approximantes qui font ressortir au mieux les différences et les analogies qu'offre la nature.

Mais il est une autre façon de faire face à l'arbitraire, c'est de multiplier les perspectives. S'il s'agit moins de faire avec un principe que de bien faire, tous les points de vue doivent venir au concours. Le but déclaré de la minéralogie est d'assurer une connaissance complète des substances

108. Ibid., t. 57, p. 294. 
minérales; ce qui implique de convoquer, soit simultanément, soit successivement, les cinq branches de la minéralogie.

Grâce à l'utilisation du principe de composition, au détour par les règles de substitution, à la multiplication des points de vue (véritable encyclopédie de la minéralogie), Werner est en position favorable pour répondre au vœu de Hardenberg : frayer des chemins, des passages de la caractéristique à l'essence. Werner lit les signes dispersés à la surface, il lit dans les minéraux à livre ouvert. Car les apparences sont les interprètes de la composition ${ }^{109}$; ce qui suppose, à l'évidence, des lois d'entreexpression.

Il est non seulement capable de reconnaître les minéraux selon l'aspect, mais de passer outre : de l'autre côté de la reconnaissance pour atteindre à la connaissance. De sorte que Werner est tout à fait capable de répondre d'avance, d'annoncer, par exemple, à l'avance la quantité de métal que chaque minéral peut donner.

En ce sens, un exemple majeur vaut d'être cité : lors de la visite de Werner au laboratoire de l'École des mines de Paris (1803), alors que divers échantillons de minerai de fer allaient être soumis à l'essai docimastique, Werner, après les avoir pris en main et les avoir examinés longuement, annonça la quantité de métal que chacun donnerait. Les résultats des essais furent, à peu de choses près, conformes à l'indication donnée ${ }^{110}$.

Seule une longue pratique des règles de l'entre-expression permet de soupçonner l'essence. Ce que Werner met ici en cuvre, c'est une stratégie, pré-analytique, du soupçon qu'autorisent le regard, le sens divinatoires (Der divinatorische Sinn, Blick) ${ }^{111}$. Ce que la prise en main autorise et annonce, l'analyse permet ensuite de le confirmer, aux inexactitudes près.

Point de passage et d'échange entre les mathématiques et la minéralogie, la Bergakademie de Freiberg a été le lieu d'un transfert culturel entre la France et l'Allemagne.

Les témoins privilégiés de ce transfert, le poète romantique Friedrich von Hardenberg-Novalis et le futur ingénieur en chef des mines JeanFrançois d'Aubuisson, ont, chacun à leur façon, tenté d'intégrer, l'un la mathématique de l'infini au sein du monde des circonstances, l'autre la gravité de la terre au sein du calcul.

Les enjeux, de part et d'autre, sont d'importance : ici, il s'agit, pour optimiser l'exploitation des salines saxonnes, de recueillir au mieux la

109. Journal de physique..., frimaire an $\mathrm{X}, \mathrm{p} .453-454$.

110. Annales de chimie, t. 69, p. 226.

111. HKA, IV, p. 298-299. 
donne des éléments (soleil et vents, en particulier), d'encadrer l'aléa des "météores"; là, en mettant en contact la minéralogie française et la minéralogie allemande, de conserver une branche importante d'industrie à la France et de tirer le parti le plus avantageux des richesses minérales du territoire.

Cette intégration croisée n'a pu s'accomplir d'un coup; elle a requis, comme vecteurs de ce transfert, certaines lignes de fracture au cœur même de l'écriture. En effet, le monde, tel qu'il se présente à Hardenberg, est mixte : jamais simple, toujours composé, lacunaire parfois. C'est au soir de la vie (juin 1800) que le parcours s'achève en une tentative de cartographie ; car lever le terrain est une autre façon de lever les obstacles et les obstructions. À cette fin, le pas en avant est décisif. Mais œ pas est-il toujours possible ? Et à quelles conditions? Qu'est-ce qui le retient et le suspend parfois?

Le réel en parts mêlées peut être saisi, au choix, par l'empirisme (actif) ou le calcul. L'un est un exercice de longue patience, l'autre une saisie qui peut être fulgurante ; l'un fraye les chemins, que l'autre surplombe d'un regard. Chacun, d'ailleurs, requiert une écriture propre : l'une retient, dans son écriture même, les traces de son cheminement; l'autre efface, au fur et à mesure où elle avance, toute trace de son opérer.

D'un côté, une écriture à effet de mémoire; de l'autre, une écriture sur fond d'oubli. Mais entre les deux s'intercale la Tätigkeit qui installe et dévoile un monde.

Daniel LANCEREAU, C.R.D.H.M. (C.N.R.S., Poitiers). 